\title{
Qualitative Analysis of Some Libration Points in the Restricted Four-body Problem
}

\author{
A. Ligęza, H. Żołądek
}

We consider the situation where three heavy gravitational bodies form the Lagrange configuration rotating in a fixed plane and the fourth body of negligible mass moves in this plane. We present three cases of so-called libration points and we study their stability using linear approximation and KAM theory. In some situations we prove the Lyapunov stability for generic values of some parameter of the problem.

Keywords: Lagrange configuration, libration point, stability

\section{Introduction}

One of spectacular applications of the Kolmogorov-Arnold-Moser (KAM) theory was the proof of the Lyapunov stability of the triangular libration points in the restricted three-body problem.

Recall that in that problem one body $A$ (an asteroid of negligible mass) moves in a gravitational field generated by two heavy bodies $S$ (from the Sun) and $J$ (from the Jupiter) which rotate with constant angular velocity along two circles in a plane which contains $A$. In the coordinate frame in which the heavy bodies rest, one gets an autonomous Hamiltonian system for the motion of $A$. The latter system has five equilibrium points, three of which $L_{1}, L_{2}$ and $L_{3}$ (called the collinear libration points) correspond to positions along the line joining $S$

Received August 04, 2020

Accepted August 22, 2021

Supported by Polish NCN OPUS grant 2017/B/ST1/00931

Adam Ligȩza

al320166@mimuw. edu.pl

Institute of Mathematics, University of Warsaw,

ul. Banacha 2, 02-097 Warsaw, Poland

Henryk Żołądek

zoladek@mimuw . edu .pl

Institute of Mathematics, University of Warsaw,

ul. Banacha 2, 02-097 Warsaw, Poland

RUSSIAN JOURNAL OF NONLINEAR DYNAMICS, 2021, 17(4), 369-390 
and $J$, and the other two $L_{4}$ and $L_{5}$ (called the triangular libration points) correspond to positions at the vertices of two equilateral triangles with base $S J$. The points $L_{1,2,3}$ are linearly unstable, while the points $L_{4,5}$ have eigenvalues $\pm i \omega_{1}, \pm i \omega_{2}$ (for some range of the parameter $\mu=\operatorname{mass}(J) /(\operatorname{mass}(S)+\operatorname{mass}(J)))$. The reduction to the Birkhoff normal form of degree 4 , while avoiding resonances of order 3 and 4 , leads to a Hamiltonian function of the form $H_{0}+H_{1}$, where $H_{1}$ is small and $H_{0}$ is a Hamiltonian of a completely integrable system, as in the KAM theorem (see $[2,3,17,23,28,30]$ ). The nondegeneracy condition from that theorem is satisfied by all values of $\mu$, but one computed by A. Deprit and A. Deprit-Bartholomé [15]. The KAM theorem states that on the 3 -dimensional level hypersurfaces $\left\{H_{0}+H_{1}=\right.$ const $\}$ there exist a lot of invariant tori, each dividing the latter hypersurface into its invariant interior and its exterior.

The aim of this paper is to study an analogous problem when $A$ is moving in a gravitational field generated by three heavy bodies $S, J$ and $P$ (a planet) forming the so-called Lagrangian configuration. The latter means that they lie in vertices of an equilateral triangle which rotates with constant angular velocity in a fixed plane which contains $A$. The passage to an autonomous system is the same as above, but the equations for the libration points are highly complex and without explicit solutions. But some such points can be found after imposing suitable restrictions on the parameters of the problem and applying a perturbation analysis. We consider three types of such libration points.

In the first case the relative mass $\nu$ of $P$ is small and the libration points lie near $P$. There are four of them and they are expanded in power series of $\nu^{1 / 3}: L_{1,2}^{ \pm}(\nu)=P \pm \nu^{1 / 2} Q_{1,2}+\ldots$. Here we prove that, for the relative mass $\mu$ of $J$ from some interval and generic, two of the libration points, say $L_{2}^{ \pm}$, are Lyapunov stable, while the other two are always unstable, see Theorem 1 and Remark 2 (about earlier research).

In the second case we assume that the masses of $S$ and of $J$ are equal and we study libration points on the symmetry line of the triangle $\triangle S J P$ through $P$. Here we do not have such a rigorous result, because some analysis is based on numerical computations. We have found that generically there are either two libration points $L_{1,2}$ or four libration points $L_{1, \ldots, 4}$ on the symmetry line. One of them $L_{2}$ or three of them $L_{2,3,4}$ are always unstable, but $L_{1}$ is generically stable when the parameter $\mu$ (the relative mass of $J$ ) lies in some small interval near $\mu=0$, see Theorem 2 and Remark 3.

In the third case we assume that the masses of $J$ and of $P$ are small and we look for libration points near the circle with center at $S$ and of radius $|S J|$. Here we prove the generic Lyapunov stability in some situations, see Theorem 3.

As one could expect, the restricted four body problem has attracted other investigators.

Firstly, some results on the four-body problem were presented by V. Brumberg [10]. There the so-called permanent configurations of four bodies are considered; those configurations are such that the ratios of distances between the bodies are constant. The following situations were described: all bodies on a line (6 solutions); the bodies not in a plane (only the unstable tetrahedron configuration); all bodies in a plane (the unstable square, the unstable rhombus, the unstable equilateral triangle with the fourth body at the center, the isosceles trapezoid, the rhombohedron and the symmetric restricted four-body problem (see also Remark 3 below); the Orion configuration.

K. Howell and D. Spencer [19] studied the so-called periodic halo orbits which bifurcate from the collinear libration points in the restricted three-body problem $(S-J-A)$ when the fourth body $P$ appears far away from the $S-J$ system.

In [4] the libration points in the restricted four-body problem were studied numerically. The authors write: "It was found that if the primary bodies are equal then the dynamical model 
admits four collinear and six non-collinear equilibria. All the equilibrium points, in this case, are unstable. If two primary bodies are equal then the problem has eight or ten equilibrium solutions and some of them are stable for limited intervals of the parameters. If the three primaries are unequal then [...] the problem, in this case, has eight or ten equilibria and some of them are stable". But we have the earlier paper [1], where 8 or 9 or 10 such points were found, but it was not known if one of the nine points can be continued to the case with positive mass.

In [5] the authors studied numerically families of periodic orbits. There are ten families, each indexed by the value of the total energy of the asteroid (called the Jacobian constant).

In [14] the $3+1$ problem with one dominant mass was studied. The authors obtained two different classes of such central configurations. In the symmetric case there is numerical evidence that the number of such central configurations varies from 5 to 7 .

In [20] the restricted four-body problem was studied in the symmetric case (it agrees with other results) and in the case where $A$ lies outside the Lagrange triangle (6 configurations). In [22] the authors use functional analytic methods and a version of Maslov index to study stability of some situations from [20].

In [12] and in [13] the situation with small mass $\nu$ of the planet was considered. The results partially agree with ours from Section 3 (see Remark 2). In [13] also the Poincaré return map (to a suitably chosen section) was studied numerically.

We also recommend the works [18] and [29] about the Birkhoff normal form.

Nevertheless, it seems that our work is one of the first where KAM theory is effectively used in the four-body problem.

\section{General statements}

In this section we present some formulas which are used in the subsequent sections. Some of them are classical (we provide them for completeness) and others are relatively new.

\subsection{Hamiltonian of the problem}

By choosing the units of mass, length and time we can assume that the sum of masses of the three heavy bodies, the angular velocity and the gravitational constant are equal to 1 . Then the length of the sides of the Lagrangian triangle is also equal to 1.

So, the Hamiltonian function associated with the movement of the asteroid $A=(x, y)$ takes the form

$$
\frac{1}{2}\left(p_{x}^{2}+p_{y}^{2}\right)-\frac{1-\mu-\nu}{\rho_{S}}-\frac{\mu}{\rho_{J}}-\frac{\nu}{\rho_{P}}
$$

where $1-\mu-\nu, \mu, \nu$ and $\rho_{S}, \rho_{J}, \rho_{P}$ are the corresponding masses and distances to $A$. This Hamiltonian depends directly on time.

Assuming the center of mass at the origin, one applies the rotation to the coordinates $(x, y)$ and to the momenta $\left(p_{x}, p_{y}\right)$. In the new coordinates the bodies $S, J, P$ rest and $A$ moves accordingly to the Hamiltonian system with the following time-independent Hamiltonian $H=$ $=\frac{1}{2}\left(p_{x}+y\right)^{2}+\frac{1}{2}\left(p_{y}-x\right)^{2}-W(x, y)$, where $W=\frac{1}{2}\left(x^{2}+y^{2}\right)+(1-\mu-\nu) / \rho_{S}+\mu / \rho_{J}+\nu / \rho_{P}$ is a "minus effective potential".

For some simplicity reasons it is useful to put $S$ at $(0,0), J$ at $(1,0)$ and $P$ at $(1 / 2, \sqrt{3} / 2)$. Then the above $H$ and $W$ should be replaced with

$$
H=\frac{1}{2}\left(p_{x}+y-y_{c}\right)^{2}+\frac{1}{2}\left(p_{y}-x+x_{c}\right)^{2}-W(x, y),
$$




$$
\begin{aligned}
& W=\frac{1}{2}\left(x-x_{c}\right)^{2}+\frac{1}{2}\left(y-y_{c}\right)^{2}+\frac{1-\mu-\nu}{\rho_{S}}+\frac{\mu}{\rho_{J}}+\frac{\nu}{\rho_{P}}, \\
& x_{c}=\mu+\frac{\nu}{2}, \quad y_{c}=\frac{\sqrt{3}}{2} \nu .
\end{aligned}
$$

Recall also that

$$
\rho_{S}^{2}=x^{2}+y^{2}, \quad \rho_{J}^{2}=(x-1)^{2}+y^{2}, \quad \rho_{P}^{2}=\left(x-\frac{1}{2}\right)^{2}+\left(y-\frac{\sqrt{3}}{2}\right)^{2} .
$$

\subsection{Libration points}

The equilibrium points of the corresponding Hamiltonian systems are the critical points of $H$, i. e., $p_{x}=y_{c}-y_{j}, p_{y}=x_{j}-x_{c}$, where the libration points $L_{j}=\left(x_{j}, y_{j}\right)$ are the critical points of the function $W$.

With such a point we associate the local (affine) coordinates

$$
q_{1}=x-x_{j}, \quad q_{2}=y-y_{j}, \quad p_{1}=p_{x}+y_{j}-y_{c}, \quad p_{2}=p_{y}-x_{j}+x_{c} .
$$

Then the Taylor expansion of $H$ at such a critical point takes the form

$$
H=\text { const }+H_{2}(p, q)+H_{3}(q)+H_{4}(q)+\ldots,
$$

where

$$
H_{2}=\frac{1}{2}\left(p_{1}+q_{2}\right)^{2}+\frac{1}{2}\left(p_{2}-q_{1}\right)^{2}-\frac{a}{2} q_{1}^{2}-b q_{1} q_{2}-\frac{c}{2} q_{2}^{2},
$$

and $H_{3,4}$ are homogeneous polynomials of degree 3,4 .

To find expressions for $H_{3}$ and $H_{4}$ we will use the following elementary

Lemma 1. Consider the function $f(q)=|Q+q|^{-1}$ for fixed $Q \in \mathbb{R}^{2} \backslash 0$ and small $q \in \mathbb{R}^{2}$. We have

$$
\begin{array}{r}
f(q)=\frac{1}{|Q|}-\frac{(Q, q)}{|Q|^{3}}+\frac{1}{2|Q|^{5}}\left\{3(Q, q)^{2}-|Q|^{2}|q|^{2}\right\}+\frac{(Q, q)}{2|Q|^{7}}\left\{3|Q|^{2}|q|^{2}-5(Q, q)^{2}\right\}+ \\
+\frac{1}{8|Q|^{9}}\left\{3|Q|^{4}|q|^{4}-60|Q|^{2}|q|^{2}(Q, q)^{2}+35(Q, q)^{4}\right\}+\ldots
\end{array}
$$

\subsection{Linear approximation}

Lemma 2. The characteristic polynomial of the linearization matrix of the Hamiltonian system at such a critical point takes the form

$$
P(\lambda)=\lambda^{4}+a_{2} \lambda^{2}+a_{4}
$$

where

$$
a_{2}=4-a-c, \quad a_{4}=a c-b^{2} .
$$

Lemma 3. The open necessary and sufficient conditions for the Lyapunov linear stability of such an equilibrium point are

$$
a_{2}>0, \quad a_{4}>0, \quad \Delta=a_{2}^{2}-4 a_{4}>0
$$


REMARK 1 . In the restricted three-body problem $(\nu=0)$, for the triangular libration points corresponding to $P$, we have

$$
a=\frac{3}{4}, \quad b=\zeta=: \frac{3 \sqrt{3}(1-2 \mu)}{4}, \quad c=\frac{9}{4} .
$$

Hence, $P(\lambda)=\lambda^{4}+\lambda^{2}+27 / 16-\zeta^{2}$ and $\Delta=4 \zeta^{2}-23 / 4$. So, the conditions from Lemma 3 hold for $0<\mu<\frac{1}{2}(1-\sqrt{23 / 27})$.

\subsection{Birkhoff normal form and isoenergetic nondegeneracy condition}

Assuming that the linear part of the Hamiltonian vector field $X_{H}=\{\cdot, H\}$ has pure imaginary eigenvalues $\pm i \omega_{1}, \pm i \omega_{2}$ we can choose complex linear coordinates $z_{1}, \bar{z}_{1}, z_{2}, \bar{z}_{2}$ such that

$$
\dot{z}_{j}=\left\{z_{j}, H\right\}=i \omega_{j} z_{j}+\ldots, \quad \dot{\bar{z}}_{j}=-i \omega_{j} \bar{z}_{j}+\ldots, \quad j=1,2 .
$$

(Above $\{\cdot, \cdot\}$ denotes the standard Poisson bracket.) Then we can write

$$
H_{2}=i \omega_{1} \frac{\left|z_{1}\right|^{2}}{\kappa_{1}}+i \omega_{2} \frac{\left|z_{2}\right|^{2}}{\kappa_{2}}
$$

where $\kappa_{j}=\left\{z_{j}, \bar{z}_{j}\right\}$ and $\left|\omega_{2}\right| /\left|\omega_{1}\right| \leqslant 1$. The above notations are taken from [8], but, when $z_{j}=q_{j}+i p_{j}, \bar{z}_{j}=q_{j}-i p_{j}$ and $\left\{q_{j}, p_{k}\right\}=\delta_{j k},\left\{q_{j}, q_{k}\right\}=\left\{p_{j}, p_{k}\right\}=0$, then $\kappa_{j}=-2 i$ and

$$
H_{2}=-\frac{1}{2} \omega_{1}\left(q_{1}^{2}+p_{1}^{2}\right)-\frac{1}{2} \omega_{2}\left(q_{2}^{2}+p_{2}^{2}\right)=-\omega_{1} I_{1}-\omega_{2} I_{2} .
$$

The fourth-order Birkhoff normal form [9, 23] in the above situation is a symplectic change of variables

$$
Z_{j}=z_{j}+\ldots, \quad \bar{Z}_{j}=\bar{z}_{j}+\ldots
$$

such that

$$
H(z, \bar{z})=H^{\operatorname{Bir}}(Z, \bar{Z})+\ldots=-\sum \omega_{j} J_{j}+\sum D_{j k} J_{j} J_{k}+\ldots
$$

where $J_{k}=i\left|Z_{k}\right|^{2} /\left\{Z_{k}, \bar{Z}_{k}\right\}=i\left|Z_{k}\right|^{2} / \kappa_{k}$. We recall also that this formula holds in the absence of low-order resonances, i. e., $\left|\omega_{1}\right| /\left|\omega_{2}\right| \neq 1,2,3$.

The quartic polynomial $H^{\text {Bir }}$ is a Hamiltonian function of a completely integrable Hamiltonian system with a family $\left\{J_{1}=c_{1}, J_{2}=c_{2}\right\}$ of invariant tori. Each such torus, parametrized by the angles $\varphi_{1}, \varphi_{2}$, supports a motion defined by the equations

$$
\dot{\varphi}_{j}=\omega_{j}(J)=\frac{\partial H^{\mathrm{Bir}}}{\partial J_{j}}, \quad j=1,2,
$$

which is either periodic or quasi-periodic, depending on the ratio $\omega_{2}(J) / \omega_{1}(J)$ of the frequencies.

The isoenergetic nondegeneracy condition, needed in the KAM theorem, says that the above ratio varies regularly when restricted to a fixed energy hypersurface $\left\{H^{\mathrm{Bir}}=h\right\}$. Explicitly, it says that the following determinant:

$$
\operatorname{det}:=\left|\begin{array}{cc}
\partial^{2} H^{\mathrm{Bir}} / \partial J_{j} \partial J_{k} & \partial H^{\mathrm{Bir}} / \partial J_{k} \\
\partial H^{\mathrm{Bir}} / \partial J_{j} & 0
\end{array}\right|=-2 \omega_{2}^{2} D_{11}+2 \omega_{1} \omega_{2} D_{12}-2 \omega_{1}^{2} D_{22},
$$

evaluated at $J_{1}=J_{2}=0$, is nonzero.

Recall that, in the case of the restricted three-body problem, the above determinant was computed in [15]. In [8] its geometric derivation was proposed using some monodromy properties of $D_{j k}$ and treating det as algebraic functions of the parameter $\mu$, i. e., in $\mathbb{C}$. 


\subsection{Bifurcations near the $0: 1$ resonance}

In this paper we encounter situations where $a_{4} \rightarrow 0$ in Lemma 2 as some parameter tends to a bifurcational value. This means the $0: 1$ resonance. The corresponding bifurcations were analyzed in $[8] .^{1}$

The quadratic part of the Hamiltonian function can be reduced to

$$
H^{(2)}(z, \widetilde{z}, u, v)=\frac{\lambda}{\kappa}|z|^{2}-\frac{1}{2} u^{2}
$$

where $\lambda=i \omega_{1}, z=z_{1}, \kappa=\{z, \bar{z}\}$ (as before) and $u, v$ are coordinates with $\{u, v\}=1$ (see $[8$, Eq. (3.1)]).

Assume the following expansion of the Hamiltonian:

$$
\begin{aligned}
H=H^{(2)} & +A_{1} v^{3}+A_{2} v|z|^{2}+A_{3} v^{4}+A_{4} v^{2}|z|^{2}+A_{5}|z|^{4}+ \\
& +\left(k_{30} u^{3}+k_{21} u^{2} v+k_{12} u v^{2}+l u|z|^{2}\right)+z M+\bar{z} \bar{M}+z^{2} N+\bar{z}^{2} \bar{N}+T+\ldots,
\end{aligned}
$$

where $M=m_{20} u^{2}+m_{11} u v+m_{02} v^{2}, N=n_{1} u+n_{2} v, T=\sum_{j+k=3} t_{j k} z^{j} \bar{z}^{k}$ and the dots contain inessential quartic and higher-order terms (see [8, Eq. (3.2)]).

After applying a concrete symplectic change $u=U+\ldots$, etc., we arrive at the following analogue of the Birkhoff normal form:

$$
H^{\text {Nor }}=\frac{\lambda}{\kappa}|Z|^{2}-\frac{1}{2} U^{2}+B_{1} V^{3}+B_{2} V|Z|^{2}+B_{3} V^{4}+B_{4} V^{2}|Z|^{2}+B_{5}|Z|^{4}+\ldots,
$$

where

$$
\begin{gathered}
B_{1}=A_{1}, \quad B_{2}=A_{2}, \\
B_{3}=A_{3}+\frac{1}{2} k_{12}^{2}-\frac{\kappa}{\lambda}\left|m_{02}\right|^{2}-\frac{3}{2} A_{1} k_{21}-2 A_{2} \operatorname{Re}\left(m_{02}\right), \\
B_{4}=A_{4}+k_{12} l-3 \frac{\kappa}{\lambda}\left|n_{2}\right|^{2}-\frac{1}{2} A_{2} k_{21}-4 \frac{\kappa}{\lambda} \operatorname{Re}\left(t_{21} m_{02}\right), \\
B_{5}=A_{5}+\frac{1}{2} l^{2}-3 \frac{\kappa}{\lambda}\left(\left|t_{03}\right|^{2}+\left|t_{21}\right|^{2}\right)
\end{gathered}
$$

(see [8, Eqs. (3.4)-(3.5)]).

Consider now a deformation $H_{\varepsilon}, \varepsilon \in(\mathbb{C}, 0)$, of Hamiltonian (2.11) such that the origin $u=v=z=\widetilde{z}=0$ is critical and for $\varepsilon \neq 0$ the corresponding matrix $A_{\varepsilon}$ is nondegenerate. The natural deformation of the Jordan cell is $\left(\begin{array}{cc}0 & \varepsilon \\ -1 & 0\end{array}\right)$. The whole family $H_{\varepsilon}$ can be reduced to the following normal form:

$$
H_{\varepsilon}^{\mathrm{Nor}}=\frac{\lambda}{\kappa}|Z|^{2}-\frac{1}{2}\left(U^{2}+\varepsilon V^{2}\right)+B_{1} V^{3}+B_{2} V|Z|^{2}+B_{3} V^{4}+B_{4} V^{2}|Z|^{2}+B_{5}|Z|^{4}+\ldots,
$$

where the coefficients $\lambda=\lambda(\varepsilon), \kappa=\kappa(\varepsilon), B_{j}=B_{j}(\varepsilon)$ depend analytically on the parameter and $B_{j}(0)$ are given in Eqs. (2.13).

\footnotetext{
${ }^{1}$ In [8] bifurcations at other resonances were considered. That analysis has led to Deprit's formula. Also, such bifurcations (and corresponding monodromy maps) were considered: in [11] (general theory), in $[6,16,27,31]$ (1:1 resonance) and in [26] (2:1 resonance).
} 
It turns out that the Birkhoff normal form associated with the latter Hamiltonian is such that

$$
\begin{aligned}
D_{11} & =\kappa^{2}\left(-\frac{B_{2}^{2}}{2 \varepsilon}+B_{5}\right), \\
D_{12} & =\frac{i \kappa}{\sqrt{\varepsilon}}\left(-\frac{3 B_{1} B_{2}}{\varepsilon}+B_{4}\right), \\
D_{22} & =\frac{15 B_{1}^{2}}{4 \varepsilon^{2}}-\frac{3 B_{3}}{2 \varepsilon}
\end{aligned}
$$

(see [8, Eqs. (3.8)]).

For more information about the phase portrait in the $0: 1$ resonance (e.g., periodic solutions) we refer the reader to the works $[24,25]$ of A. Markeev.

\subsection{Some calculations for the $0: 1$ resonance}

In the following three sections we encounter situations with the $0: 1$ resonance, but with different data. Therefore, it is reasonable to get some general formulas for this case.

Assume that we have the Hamiltonian function (2.6) such that

$$
a+c=3, \quad a c=b^{2} .
$$

Thus, we have the linear system

$$
\dot{q}_{1}=p_{1}+q_{2}, \quad \dot{p}_{1}=(a-1) q_{1}+b q_{2}+p_{2}, \quad \dot{q}_{2}=p_{2}-q_{1}, \quad \dot{p}_{2}=b q_{1}-p_{1}+(c-1) q_{2},
$$

with the eigenvalues $\lambda= \pm i$ and $\lambda=0$ (double).

Then the variable $z$ from Eq. (2.10), the eigenfunction with the eigenvalue $i$, becomes

$$
z=(a-1-i b) q_{1}+(-b+2 i) p_{1}+(b+(a-1) i) q_{2}+(a+1) p_{2},
$$

with $\kappa=\{z, \bar{z}\}=2(a+1) i$. Next, the function

$$
\widetilde{u}=a q_{1}-b p_{1}+b q_{2}+b a p_{2}
$$

is invariant (eigenfunction with zero eigenvalue) and the function

$$
\widetilde{v}=-b q_{1}+2 p_{1}+(a-2) q_{2}
$$

satisfies $d \widetilde{v} / d t=\widetilde{u}$. But $\{\widetilde{u}, \widetilde{v}\}=a$; so we put

$$
u=\frac{\widetilde{u}}{\sqrt{a}}, \quad v=\frac{\widetilde{v}}{\sqrt{a}}
$$

in Eq. (2.10). We have

$$
H_{2}=\frac{1}{2(a+1)}|z|^{2}-\frac{1}{2} u^{2}
$$

In the analogue of the Birkhoff normal form $H^{\text {Nor }}$ (in Eq. (2.12)) and in the Birkhoff normal form after perturbation (in Eqs. (2.14)), it is the coefficients $B_{1}, B_{2}$, etc. that are essential. So, we are interested in the inverse change. 
By comparison of Eqs. (2.17)-(2.19) we find that

$$
z=z_{1}+z_{2} i=\left(\widetilde{u}-q_{1}+p_{2}\right)+\left(\widetilde{v}+q_{2}\right) i
$$

thus,

$$
p_{2}=q_{1}+z_{1}-\widetilde{u}, \quad q_{2}=z_{2}-\widetilde{v} .
$$

Then Eqs. (2.18)-(2.19) lead to the system

$$
\left(\begin{array}{cc}
2 a & -b \\
-b & 2
\end{array}\right)\left(\begin{array}{l}
q_{1} \\
p_{1}
\end{array}\right)=\left(\begin{array}{c}
(a+1) \widetilde{u}+b \widetilde{v}-a z_{1}-b z_{2} \\
(a-1) \widetilde{v}+(2-a) z_{2}
\end{array}\right),
$$

where the determinant of the matrix above is $a(a+1)$. Therefore,

$$
q_{1}=\frac{1}{a(a+1)}\left\{2(a+1) \widetilde{u}+b(a+1) \widetilde{v}-2 a z_{1}-a b z_{2}\right\} .
$$

Sometimes we are interested in the dependence of the $q_{j}$ 's on $v$ only (for the coefficients $A_{1}$ and $\left.A_{3}\right)$. Then, for $z=u=0$, we get

$$
q_{1}=\sqrt{c} \cdot v, \quad q_{2}=-\frac{a}{b} q_{1} .
$$

\section{Libration points near the Planet}

We assume

$$
\nu \ll 1
$$

and $0<\mu<\frac{1}{2}$. Thus, $x_{c}=\mu+O(\nu)$ and $y_{c}=O(\nu)$.

We look for libration points near $P$. So, we assume $L=P+\nu^{\alpha} Q+\ldots, Q=(X, Y), \alpha>0{ }^{2}$

The minus effective potential (2.3), with use of the data (2.7), takes the form

$$
\begin{aligned}
W & =\text { const }+\nu^{2 \alpha}\left\{\frac{3}{8} X^{2}+\zeta X Y+\frac{9}{8} Y^{2}+\ldots\right\}+\nu^{1-\alpha} \frac{1}{R}+O(\nu), \\
R & =\sqrt{X^{2}+Y^{2}}=|Q|,
\end{aligned}
$$

where

$$
\zeta=\frac{3 \sqrt{3}}{4}(1-2 \mu)
$$

Therefore,

$$
\alpha=\frac{1}{3}
$$

and one can see that $L=L(\nu)$ can be expanded in powers of $\nu^{1 / 3}$ (a Puiseux type expansion).

${ }^{2}$ Of course, there exists a libration point near the other libration point $L_{5}$ of the restricted three-body problem. But the differential system near it is a slight perturbation of the corresponding three-body system. So, it is Lyapunov stable for $0<\mu<\frac{1}{2}(1-\sqrt{23 / 27})$ and $\mu$ separated from the resonant values and from Deprit's critical value. 
The critical points condition reads as $\nabla \Psi=0, \Psi=\frac{3}{8} X^{2}+\zeta X Y+\frac{9}{8} Y^{2}+\frac{1}{R}$. We obtain the equations

$$
\frac{3}{4} X+\zeta Y=\frac{X}{R^{3}}, \quad \zeta X+\frac{9}{4} Y=\frac{Y}{R^{3}} .
$$

Dividing them and putting $Y=\eta X$, we arrive at the equation

$$
\zeta \eta^{2}-\frac{3}{2} \eta-\zeta=0
$$

with two solutions

$$
\eta_{1}=\frac{1}{4 \zeta}\left(3+2 \sqrt{\frac{9}{4}+4 \zeta^{2}}\right), \quad \eta_{2}=\frac{1}{4 \zeta}\left(3-2 \sqrt{\frac{9}{4}+4 \zeta^{2}}\right) .
$$

These solutions correspond to four critical points $\pm Q_{1,2}=\left( \pm X_{1,2}, \pm Y_{1,2}\right)$,

$$
\begin{aligned}
& X_{1,2}=\left(1+\eta_{1,2}^{2}\right)^{-1 / 2}\left(\frac{3}{4}+\eta_{1,2} \zeta\right)^{-1 / 3} \\
& Y_{1,2}=\eta_{1,2} X_{1,2}
\end{aligned}
$$

moreover,

$$
R_{1,2}=\left(\frac{3}{4}+\eta_{1,2} \zeta\right)^{-1 / 3}
$$

To get a Taylor expansion of the function $W$ at the libration point

$$
L_{1,2}^{ \pm}(\nu) \approx P \pm \nu^{1 / 3} Q_{1,2}
$$

we put $(x, y)=L_{1,2}^{ \pm}+q, q=\left(q_{1}, q_{2}\right)$, and expand it in powers of $q$. From the regular part $W-\nu / \rho_{P}$ of $W$ we get: const + linear part $+F_{2}(q)+\ldots$ as $\nu \rightarrow 0$, where

$$
F_{2}(q)=\frac{3}{8} q_{1}^{2}+\zeta q_{1} q_{2}+\frac{9}{8} q_{2}^{2}
$$

To expand the singular part $\nu / \rho_{P}$ of $W$ we use Lemma 1 :

$$
\frac{\nu}{\rho_{P}}=\frac{\nu}{\left|\nu^{1 / 3} Q_{1,2}+q\right|}=\nu^{2 / 3} G_{0}(q)+\nu^{1 / 3} G_{1}(q)+G_{2}(q)+\ldots
$$

where

$$
\begin{aligned}
G_{2} & =\frac{1}{2 R_{1,2}^{5}}\left\{3\left(Q_{1,2}, q\right)^{2}-R_{1,2}^{2}|q|^{2}\right\} \\
G_{3} & = \pm \frac{\left(Q_{1,2}, q\right)}{2 R_{1,2}^{7}}\left\{3 R_{1,2}^{2}|q|^{2}-5\left(Q_{1,2}, q\right)^{2}\right\} \\
G_{4} & =\frac{1}{8 R_{1,2}^{9}}\left\{3 R_{1,2}^{4}|q|^{4}-60 R_{1,2}^{2}|q|^{2}\left(Q_{1,2}, q\right)^{2}+35\left(Q_{1,2}, q\right)^{4}\right\} .
\end{aligned}
$$

Therefore, we arrive at the following result. 
Lemma 4. In the Taylor expansion $W=W_{0}+W_{1}(q)+\ldots$ at $L_{1,2}^{ \pm}$, as $\nu \rightarrow 0$, we have

$$
\begin{aligned}
& W_{2}=F_{2}+G_{2}+\ldots, \\
& W_{3}=\nu^{-1 / 3} G_{3}+\ldots, \\
& W_{4}=\nu^{-2 / 3} G_{4}+\ldots
\end{aligned}
$$

In particular, in Lemma 2 we have

$$
\begin{aligned}
a & =\frac{3}{4}+3 X_{1,2}^{2} R_{1,2}^{-5}-R_{1,2}^{-3}+\ldots, \\
b & =\zeta+3 X_{1,2} Y_{1,2} R_{1,2}^{-5}+\ldots, \\
c & =\frac{9}{4}+3 Y_{1,2}^{2} R_{1,2}^{-5}-R_{1,2}^{-3}+\ldots
\end{aligned}
$$

Theorem 1. The libration points $L_{1}^{ \pm}$are unstable and, for $\mu^{\prime}$ 's from the interval $\left(0, \mu_{0}\right)$, where $\mu_{0} \approx 0.011942$, deprived of a finite number of points, the libration points $L_{2}^{ \pm}$are Lyapunov stable (for $\mu>\mu_{0}$ they are unstable).

Proof. From Eq. (3.3) we can express $\zeta$ as a function of $\eta$ :

$$
\zeta=\frac{3}{2} \frac{\eta}{\eta^{2}-1}
$$

Since $0<\zeta<3 \sqrt{3} / 4$, we consider the latter function in two intervals

$$
I_{1}=(\sqrt{3}, \infty) \quad \text { and } \quad I_{2}=\left(-\frac{1}{\sqrt{3}}, 0\right)
$$

thus, $\eta_{1} \in I_{1}$ and $\eta_{2} \in I_{2}$. We have

$$
\begin{aligned}
R^{-3} & =\frac{3}{4}+\eta \zeta=\frac{3}{4} \frac{1-3 \eta^{2}}{1-\eta^{2}} \\
X & = \pm \frac{R}{\sqrt{1+\eta^{2}}} \\
Y & = \pm \frac{\eta R}{\sqrt{1+\eta^{2}}} .
\end{aligned}
$$

Next,

$$
a=-\frac{3\left(2 \eta^{4}-7 \eta^{2}+3\right)}{4\left(\eta^{4}-1\right)}, \quad b=\frac{3}{4} \eta \frac{11 \eta^{2}-1}{\eta^{4}-1}, \quad c=-\frac{3\left(-9 \eta^{4}+5 \eta^{2}+2\right)}{4\left(\eta^{4}-1\right)} .
$$

Let us compute the quantities from Lemma 3. We have

$$
a_{2}=1-R^{-3}=-\frac{1}{4} \frac{5 \eta^{2}+1}{\eta^{2}-1}
$$

Therefore, in the case of the interval $I_{1}$, we have $\eta^{2}-1>0$ and $5 \eta^{2}+1>0$; so, $a_{2}<0$ and the points $L_{1}^{ \pm}=P \pm \nu^{1 / 3} Q_{1}+\ldots$ are linearly unstable. 
In the case of the interval $I_{2}$ we have $\eta^{2}<1 / 3$ and hence $a_{2}>0$. Next, for $\eta=\eta_{2} \in$ $\in(-1 / \sqrt{3}, 0)$, we have

and

$$
a_{4}=\frac{27}{8} \frac{\left(1-3 \eta^{2}\right)\left(\eta^{2}+1\right)}{\left(\eta^{2}-1\right)^{2}}>0
$$

$$
\Delta=\frac{673 \eta^{4}+442 \eta^{2}-215}{16\left(\eta^{2}-1\right)^{2}} .
$$

Above the polynomial $673 \eta^{4}+442 \eta^{2}-215$, as a function of $\eta^{2}$ is monotone increasing and has unique zero corresponding to $\eta_{0}=-\sqrt{(192 \sqrt{21}-442) / 1346} \approx-0.570350>-1 / \sqrt{3} \approx$ $\approx-0.57735$. It corresponds to $\zeta_{0}=\frac{1}{100} \sqrt{\frac{96}{673} \sqrt{21}-\frac{221}{673}}(16 \sqrt{21}+149) \approx 1.268$ and

$$
\mu_{0}=\frac{1}{2}\left(1-\frac{4}{3 \sqrt{3}} \zeta_{0}\right)=\frac{1}{2}-\left(\frac{8}{75} \sqrt{7}+\frac{149}{450} \sqrt{3}\right) \sqrt{\frac{96}{673} \sqrt{21}-\frac{221}{673}} .
$$

We have $\Delta>0$ for $\eta^{2}>\eta_{0}^{2}$, i. e., for $0<\mu<\mu_{0}$.

Linear stability is not sufficient for the genuine Lyapunov stability. As in the restricted three-body problem, one has to use KAM theory.

Of course, firstly one has to reduce the quartic part of the Taylor expansion of the Hamiltonian to the Birkhoff normal form; for this aim one has to exclude the values of the parameter $\mu$ corresponding to the resonances of order 3 and 4 . Since $a_{2}, a_{4}$ and $\Delta$ are algebraic functions of $\mu$, there are only finitely many such values.

Analogously, we have to exclude the values of $\mu$ corresponding to the violation of the isoenergetic nondegeneracy condition det $\neq 0$ from Section 2.4. But, for this we should know that $\operatorname{det}(\mu) \not \equiv 0$, i. e., as a function of $\mu$. We do not compute the function $\operatorname{det}(\mu)$ explicitly, we only study its behavior near some bifurcation point; it was the way chosen by A. Leontovich [21] in the three-body problem (before the explicit formula appeared in the literature [15]).

This bifurcation point corresponds to $\mu=0$, i.e., $\zeta=3 \sqrt{3} / 4$ and $\eta=-1 / \sqrt{3}$. Indeed, as $\mu \rightarrow 0^{+}$we have $\mu \approx \frac{1}{2}(1+\sqrt{3} \eta)$,

$$
R^{-3} \approx \frac{9}{2} \mu, \quad \pm X \approx \pm \frac{1}{2} \sqrt{3} R, \quad \pm Y \approx \mp \frac{1}{2} R
$$

of course, we should remember that $\operatorname{mass}(A) \ll \nu=\operatorname{mass}(P) \ll \mu=\operatorname{mass}(J)$. Hence, in Lemma 4, we have

$$
a \approx \frac{3}{4}+\frac{45}{8} \mu, \quad b \approx \frac{3}{4} \sqrt{3}-\frac{27}{8} \sqrt{3} \mu, \quad c \approx \frac{9}{4}-\frac{9}{8} \mu .
$$

We see that $a_{2} \rightarrow 1$ and $a_{4} \rightarrow 0$ in Lemma 2. Therefore, we deal with the $0: 1$ resonance and with the corresponding bifurcations described in Section 2.5. Moreover, conditions (2.16) from Section 2.6 are satisfied and hence the eigenvalues are $\pm i$ and 0 (double).

The corresponding functions $z, u, v$ from Eqs. (2.17)-(2.20) are the following:

$$
\begin{aligned}
& z=\frac{1}{4}\left\{-(1+3 \sqrt{3} i) q_{1}+(-3 \sqrt{3}+8 i) p_{1}+(3 \sqrt{3}-i) q_{2}+7 p_{2}\right\} \\
& u=\frac{\sqrt{3}}{2}\left\{q_{1}-\sqrt{3} p_{1}+\sqrt{3} q_{2}+p_{2}\right\} \\
& v=\frac{1}{2 \sqrt{3}}\left\{-3 \sqrt{3} q_{1}+8 p_{1}-5 q_{2}\right\} .
\end{aligned}
$$


In the inverse change it is the dependence of the variables $q_{j}$ on $v$ that is important to us. By Eqs. (2.22)-(2.23), for $z=u=0$, we have

$$
q_{1}=\frac{3}{2} v,\left.\quad q_{2}\right|_{z=x=0}=-\frac{\sqrt{3}}{2} v .
$$

Therefore, $H_{2}=\left.H_{2}\right|_{\mu=0}-\frac{27 \mu}{2} v^{2}+\ldots$; so, we put

$$
\varepsilon=27 \mu
$$

in Eq. (2.14).

Next, recall that the cubic part of $H$ is $H_{3}=-\nu^{-1 / 3} G_{3} \sim O\left(\nu^{-1 / 3} \mu^{4 / 3}\right)$ and the quartic part is $H_{4}=-\nu^{-2 / 3} G_{4} \sim O\left(\nu^{-2 / 3} \mu^{5 / 3}\right)$. Therefore, in Eq. (2.11) we have $A_{1}, A_{2}, a, k_{21}, k_{12}, l$, $M \sim O\left(\nu^{-1 / 3} \mu^{4 / 3}\right)$ and $A_{3}, A_{4}, A_{5}, N, T \sim O\left(\nu^{-2 / 3} \mu^{5 / 3}\right)$; so, in Eq. (2.12) we have $B_{1}=A_{1}$, $B_{2}=A_{2} \sim O\left(\nu^{-1 / 3} \mu^{4 / 3}\right)$ and $B_{3}=A_{3}+\ldots, B_{4}, B_{5} \sim O\left(\nu^{-2 / 3} \mu^{5 / 3}\right)$.

It follows that in Eqs. (2.15) we have

$$
D_{11} \sim O\left(\nu^{-2 / 3} \mu^{5 / 3}\right), \quad D_{12} \sim O\left(\nu^{-2 / 3} \mu^{7 / 6}\right), \quad D_{22} \sim O\left(\nu^{-2 / 3} \mu^{2 / 3}\right) .
$$

Because $\lambda_{1} \sim O(1)$ and $\lambda_{2} \sim O\left(\mu^{1 / 2}\right)$, the leading term in the isoenergetic nondegeneracy determinant $\operatorname{det}(\mu)$ comes from $D_{22}$.

We have

$$
G_{3} \approx \pm \frac{1}{2} R^{-4}(Q, q)\left\{3|q|^{2}-5(Q, q)^{2}\right\},
$$

where $Q=\left(\frac{1}{2} \sqrt{3},-\frac{1}{2}\right),(Q, q)=\frac{1}{2} \sqrt{3} q_{1}-\frac{1}{2} q_{2} \simeq \sqrt{3} v$ and $|q|^{2}=q_{1}^{2}+q_{2}^{2} \simeq 3 v^{2}$. Since $H_{3}=$ $=-\nu^{-1 / 3} G_{3}=A_{1} v^{3}$ for $z=u=0$, we have

$$
A_{1} \approx \mp \frac{1}{2} \nu^{-1 / 3}\left(\frac{9}{2} \mu\right)^{4 / 3}\{\sqrt{3}(3 \cdot 3-5 \cdot 3)\} \approx \pm 2^{-4 / 3} 3^{25 / 6} \nu^{-1 / 3} \mu^{4 / 3}= \pm 38.604 \cdot \nu^{-1 / 3} \mu^{4 / 3} .
$$

Next,

$$
G_{4} \approx \frac{1}{8} R^{-5}\left\{3|q|^{4}-60|q|^{2}(Q, q)^{2}+35(Q, q)^{4}\right\}
$$

so

$$
\begin{aligned}
A_{3} \approx-\frac{1}{8} \nu^{-2 / 3}\left(\frac{9}{2} \mu\right)^{5 / 3}\left\{3 \cdot 3^{2}-60 \cdot 3 \cdot 3\right. & \left.+35 \cdot 3^{2}\right\} \approx \\
& \approx 11 \cdot 2^{-11 / 3} \cdot 3^{16 / 3} \nu^{-2 / 3} \mu^{5 / 3}=303.57 \cdot \nu^{-2 / 3} \mu^{5 / 3}
\end{aligned}
$$

in $H_{4}=A_{4} v^{4}$ (for $z=u=0$ ).

Finally, we find

$$
D_{22} \approx \frac{15 A_{1}^{2}}{4 \varepsilon^{2}}-\frac{3 A_{3}}{2 \varepsilon} \approx-9.199 \cdot \nu^{-2 / 3} \mu^{2 / 3} \neq 0
$$

and $\operatorname{det} \approx-\omega_{1}^{2} D_{22} \not \equiv 0 .^{3}$

${ }^{3}$ A. Leontovich in [21] also studied the Birkhoff normal form and the determinant function $\operatorname{det}(\mu)$ (in the three-body problem) near the $0: 1$ resonance. He found that det $\rightarrow \infty$, which contrasts with Deprit's formula det $=-\frac{1}{8}\left(36-541 \omega_{1}^{2} \omega_{2}^{2}+644 \omega_{1}^{4} \omega_{2}^{4}\right)\left(1-4 \omega_{1}^{2} \omega_{2}^{2}\right)^{-1}\left(4-25 \omega_{1}^{2} \omega_{2}^{2}\right)^{-1}$. The second author, i. e., H. Ż. with W. Barwicz, in a first approach to the three-body problem also repeated Leontovich's mistake; the paper [8] contains a corresponding correction. 
REMARK 2. In [12] the problem of equilibrium points in the same case was considered by D. Budzko and A. Prokopenya. There also the existence of two branches of libration points was proved. But their approach is symbolic-numerical and the result is not as explicit as ours.

J. Burgos-Garcia and M. Gidea in [13] studied the same problem. They also found that two libration points are always unstable and the other two are stable, e.g., linearly stable, provided $\mu<\widetilde{\mu}_{0}=$ $=\frac{1}{224}(112-\sqrt{2(1979+37 \sqrt{12097})}) \approx 0.0089896$. This value is different than our value $\mu_{0}$ from Theorem 1. In fact, they first performed the diagonalization of the quadratic form $\frac{3}{8} X^{2}+\zeta X Y+\frac{9}{8} Y^{2}$ from Eq. (3.3) by means of an orthogonal change of variables, and that caused an additional complication.

Finally, we note that, in the proof of the inequality det $\not \equiv 0$, one can expand the determinant function near other resonances (corresponding bifurcation formulas are given in [8]). But this would significantly enlarge the paper without improving essentially the result; the same is true in the other cases studied below.

\section{Libration points at the symmetry line}

Here we assume the symmetric configuration of the heavy bodies:

$$
\nu=1-2 \mu
$$

i. e., $m(S)=m(J)=\mu$. We look for the libration points at the symmetry line $\{x=1 / 2\}$.

With

$$
\widetilde{x}=x-\frac{1}{2}
$$

we have

$$
W=\frac{1}{2} \widetilde{x}^{2}+\frac{1}{2}\left(y-y_{c}\right)^{2}+\frac{\mu}{\rho_{S}}+\frac{\mu}{\rho_{J}}+\frac{1-2 \mu}{\rho_{P}},
$$

where $y_{c}=\sqrt{3}(1-2 \mu) / 2$. As $\widetilde{x} \rightarrow 0$ we have

$$
\begin{aligned}
\frac{1}{\rho_{S, J}} & \approx \frac{1}{\sqrt{y^{2}+1 / 4}}\left\{1 \pm \frac{\widetilde{x}}{2\left(y^{2}+\frac{1}{4}\right)}-\frac{\widetilde{x}^{2}}{2\left(y^{2}+\frac{1}{4}\right)}+\frac{3 \widetilde{x}^{2}}{8\left(y^{2}+\frac{1}{4}\right)^{2}}\right\} \\
\frac{1}{\rho_{P}} & \approx \frac{1}{\left|y-y_{P}\right|}\left\{1-\frac{\widetilde{x}^{2}}{2\left(y-y_{P}\right)^{2}}\right\}
\end{aligned}
$$

where $y_{P}=\sqrt{3} / 2$. Therefore,

$$
W=W_{0}(y)+\frac{a(y)}{2} \widetilde{x}^{2}+\ldots
$$

with

$$
a(y)=1+2 \mu \frac{\frac{1}{2}-y^{2}}{\left(y^{2}+\frac{1}{4}\right)^{5 / 2}}-\frac{1-2 \mu}{\left|y-y_{P}\right|^{3}}
$$

where $\left|y-y_{P}\right|= \pm\left(y-y_{P}\right)$.

The restriction of $W$ to the symmetry line equals

$$
W_{0}(y)=\frac{1}{2}\left(y-y_{c}\right)^{2}+\frac{2 \mu}{\sqrt{y^{2}+\frac{1}{4}}}+\frac{1-2 \mu}{\left|y-y_{P}\right|} .
$$


For each such libration point $L_{j}=\left(1 / 2, y_{j}\right)$ we have $W_{0}^{\prime}\left(y_{j}\right)=0$, i. e.,

$$
\left\{y \mp\left(y-y_{P}\right)^{-2}-y_{P}\right\}-2 \mu\left\{\frac{y}{\left(y^{2}+\frac{1}{4}\right)^{3 / 2}} \mp\left(y-y_{P}\right)^{-2}-y_{P}\right\}=0,
$$

$y=y_{j}$, and $\partial^{2} W / \partial x \partial y=0$. Hence, for the quadratic part $W_{2}=\frac{a}{2} \widetilde{x}^{2}+b \widetilde{x}\left(y-y_{j}\right)+\frac{c}{2}\left(y-y_{j}\right)^{2}$ of $W$ we have

$$
a=a\left(y_{j}\right), \quad b=0, \quad c=W_{0}^{\prime \prime}\left(y_{j}\right)
$$

where

$$
\begin{aligned}
& a=\left\{1 \mp\left(y-y_{P}\right)^{-3}\right\}+2 \mu\left\{\frac{\frac{1}{2}-y^{2}}{\left(y^{2}+\frac{1}{4}\right)^{5 / 2}} \pm\left(y-y_{P}\right)^{-3}\right\}, \\
& c=\left\{1 \pm 2\left(y-y_{P}\right)^{-3}\right\}+2 \mu\left\{\frac{2 y^{2}-\frac{1}{4}}{\left(y^{2}+\frac{1}{4}\right)^{5 / 2}} \mp 2\left(y-y_{P}\right)^{-3}\right\} .
\end{aligned}
$$

Of course, it is quite hard (impossible) to solve Eq. (4.5) for $y$ explicitly, but we can use the position $y=y_{j}$ as a parameter and express $2 \mu$ as an algebraic function of it, $2 \mu=\Phi(y)$. Then also $a$ and $c$ become functions of $y\left(=y_{j}\right)$, quite complicated. Therefore, we compute numerically $a$ and $c$ for a set of values of $y$.

We have the following statements.

1. The function $2 \mu=\Phi(y)$ takes the physically realistic values in $[0,1]$ iff either

$$
-y_{P} \leqslant y \leqslant y_{P}-1 \approx-0.13397
$$

or

$$
0 \leqslant y \leqslant y_{P}
$$

or

$$
y_{P} \leqslant y \leqslant y_{P}+1
$$

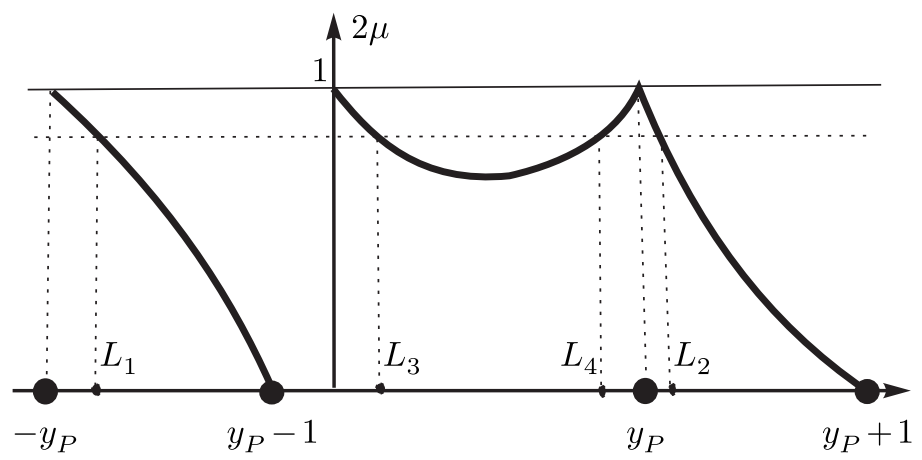

Fig. 1. Libration points

In fact, as our calculations show, the function $\Phi$ :

- decreases from 1 to 0 on the interval (4.8);

- starts with 1 and ends with 1 on the interval (4.9) (with one minimum $2 \mu_{1}$ );

- decreases from 1 to 0 on the interval (4.10). 
It is presented in Fig. 1. In particular, for each $\mu \in\left(0, \mu_{1}\right), \mu_{1} \approx 0.38$, on the symmetry line there exist two libration points $L_{j}=$ $=L_{j}(\mu), j=1,2$, and for $\mu \in\left(\mu_{1}, 1 / 2\right)$ there exist four such libration points $L_{j}=\left(1 / 2, y_{j}\right)$, $j=1,2,3,4$.

2. If $y=0$, then Eq. (4.5) gives $\mu=\frac{1}{2}$. Thus, we deal with a linear libration point of the restricted three-body problem, about which we know that it is linearly unstable. The computation of $a$ and $c$ confirms this.

3. But also the situation with $\mu \rightarrow \frac{1}{2}$ corresponds to the situation from the previous section. Near $P$ there are four libration points, two of them at the symmetry line.

Let $\widetilde{y}=y-y_{P}$. We find $2 \mu \approx 1 \mp \frac{9}{4} \widetilde{y}^{3}=1-\frac{9}{4}|\widetilde{y}|^{3}($ where $\pm=\operatorname{sign}(\widetilde{y}))$. Then $a \rightarrow-\frac{15}{4}$ and $c \rightarrow \frac{35}{4}$ So, this point is unstable, and hence the corresponding two libration points on the symmetry line are unstable.

4. Also, $\mu=\frac{1}{2}$ for $y=-y_{P}$, i. e., as in the restricted three-body problem with the lower triangular libration point. But here $\mu>\frac{1}{2}(1-\sqrt{23 / 27})$ and we are outside the stability region (see Remark 1).

5. For $y$ from the interval $\left[-y_{P}, y_{P}-1\right]$ the quantities $a$ and $c$ are positive and $a_{2}=4-$ $-a-c>0$ (see Lemma 2). But $\Delta=a_{2}^{2}-4 a_{4}$ is negative for $-y_{P} \leqslant y<y_{1}^{*} \approx-0.1357$ and is positive for $y_{1}^{*}<y \leqslant y_{P}-1$. The value $y_{1}^{*}$ corresponds to

$$
\mu=\mu_{2} \approx 0.0031 \text {. }
$$

Moreover, near the right endpoint $y_{P}-1$ we have: $2 \mu \approx 1.8666\left(y_{P}-1-y\right), a \approx-1.2681\left(y_{P}-\right.$ $-1-y)$ and hence

$$
a \approx-1.3587 \cdot \mu \text {. }
$$

6. For $0<y<1$ the quantities $a$ and $c$ change signs, but always $a_{2}<0$.

7. For $1<y<y_{P}+1$ we have $a<0$ and $c>0$; so, $a_{4}<0$.

It seems possible to demonstrate the above statements rigorously, but the proof would be rather involved. Anyway, we have the following result, part of which is proved only numerically.

Theorem 2. If $\mu_{2}<\mu<1 / 2$, then all (two or three or four) libration points $L_{j}(\mu)$ on the symmetry axis are unstable, and, if $0<\mu<\mu_{2}$, then the libration point $L_{2}(\mu)$ is unstable and the point $L_{1}(\mu)$ is Lyapunov stable, provided the parameter $\mu$ does not belong to some finite set of exceptional values.

Proof. Due to the previous analysis, it remains to apply KAM theory to the libration point $L_{1}(\mu)$ for $\mu \in\left(0, \mu_{2}\right)$. More precisely, we have to prove that the isoenergetic determinant $\operatorname{det}(\mu) \not \equiv 0$. Again we use the bifurcation theory argument.

Indeed, if $y_{1}=y_{P}-1$ (i. e., $\mu=0$ ), then $a=0$ and $c=3$ (see the first table). So, conditions (2.16) are satisfied; we have the $0: 1$ resonance with the eigenvalues $\pm i$ and 0 (double).

From Eq. (2.17) we have

$$
z=-q_{1}+2 i p_{1}-i q_{2}+p_{2}, \quad \kappa=\{z, \bar{z}\}=2 i .
$$

But, since $a=0$, we cannot use directly formulas (2.18)-(2.20). However, direct calculations give

$$
u=\sqrt{3}\left(p_{1}-q_{2}\right), \quad v=\frac{q_{1}-2 p_{2}}{\sqrt{3}} .
$$


In the inverse change we have

$$
q_{1}=-z-\bar{z}-\sqrt{3} v, \quad q_{2}=\frac{i}{2} \bar{z}-\frac{i}{2} z-\frac{2}{3} \sqrt{3} u .
$$

In particular, for $z=u=0$ we have $q_{1}=-\sqrt{3} v$ and $q_{2}=0$.

Due to Eq. (4.12), for $z=u=0$, we have $\left.H_{2} \approx H_{2}\right|_{\mu=0}-\frac{1}{2} a q_{1}^{2}$ and we can choose the small parameter as

$$
\varepsilon=3 a \approx-4.0761 \cdot \mu .
$$

Next, we have to compute the cubic and quartic terms in the expansion of the Hamiltonian, i. e., the expansion of the quantities $1 / \rho_{S, J, P}$ in Eq. (4.2). But the coefficients before $1 / \rho_{S, J}$ are small; so, we expand only $(1-2 \mu) / \rho_{P} \approx 1 / \rho_{P}$.

Since the position of $A$ with respect to $P$ is $Q_{P}=(0,-1)$, we have

$$
\left|Q_{P}\right|=1 \quad \text { and } \quad\left(Q_{P}, q\right)=-q_{2} .
$$

Thus, by Lemma 1,

$$
\begin{aligned}
H_{3}=\frac{1}{2} q_{2}\left(2 q_{2}^{2}-q_{1}^{2}\right)= & \\
=\left(-\frac{8}{9} \sqrt{3} u^{3}+\right. & \left.\sqrt{3} u v^{2}-\frac{1}{3} \sqrt{3} u|z|^{2}\right)+\left[z\left(-2 i u^{2}+2 u v+\frac{3}{4} i v^{2}\right)+\text { conj }\right]+ \\
& +\left[z^{2}\left(\frac{5}{6} \sqrt{3} u+\frac{1}{2} i \sqrt{3} v\right)+\text { conj }\right]+\frac{i}{8}\left(3 z^{3}-i\left|z^{2}\right|(z-w)-3 i \bar{z}^{3}\right) ;
\end{aligned}
$$

i. e., $A_{1}=0, A_{2}=0, k_{12}=0$ and $m_{02}=\frac{3}{4} i$ in Eq. (2.11).

Next,

$$
\left.H_{4}\right|_{\varepsilon=0}=\frac{3}{8}|q|^{4}=\frac{27}{8} v^{4}
$$

i. e., $A_{3}=\frac{27}{8}$.

We see that

$$
\begin{gathered}
B_{1}, B_{2}=O(\varepsilon), \quad B_{4}, B_{5}=O(1), \\
B_{3}=A_{3}-\frac{\kappa}{\lambda}\left|m_{02}\right|^{2}+O(\varepsilon)=\frac{9}{2}+O(\varepsilon)
\end{gathered}
$$

in Eqs. (2.13).

It follows that, in Eqs. (2.15),

$$
D_{11}=O(1), \quad D_{12}=O\left(\frac{1}{\sqrt{\varepsilon}}\right), \quad D_{22} \approx-\frac{3 B_{3}}{2 \varepsilon} .
$$

Finally, since $\lambda_{1} \approx i$ and $\lambda_{2}=O(\sqrt{\varepsilon})$, we find that

$$
\operatorname{det} \approx-2 \omega_{1}^{2} D_{22}=-\frac{81}{2 \varepsilon} \neq 0
$$

REMARK 3. From the reviewer's report of the first version of our paper we have learned that the above symmetric situation was considered also by other investigators. 
In particular, in [10] V. Brumberg describes results obtained by Meyer in 1933. Indeed, our Eq. (4.5) coincides (up to notations) with Eq. (3.11) from [10] and the linear stability of the libration point $L_{1}=$ $=L_{1}(\mu)$ is established for approximately the same range of the parameter $\mu$ as in Theorem 2 . In fact, in [10] V. Brumberg speaks about three libration points, denoted $A, B=L_{1}$ and $C=L_{2}$, but visibly $A$ is either $L_{3}$ or $L_{4}$ (compare also [20]).

In the paper [7] by B. Bardin and P. Esipov the crucial determinant (2.9) for $B=L_{1}$ is calculated numerically, as a function of $\mu$; it has one zero and a pole corresponding to $2: 1$ resonance. Moreover, for the $2: 1$ resonance one has instability and for the $3: 1$ resonance one has stability.

REMARK 4. The point $L_{1}(\mu)$ for small $\mu$ lies near the circle with center at $P$ and with radius 1 . Since the masses of $S$ and $J$ are small, this case agrees with the situation considered in the next section.

\section{Libration points near the circle}

We assume

$$
0<\mu, \nu \ll 1,
$$

but $\mu$ and $\nu$ are of the same order.

Using the standard polar coordinates $r, \varphi$ we rewrite $W$ from Eq. (2.3) as follows:

$$
W=\frac{1}{2} r^{2}+\frac{1}{r}+\mu\left(-\frac{1}{r}-x+\frac{1}{\rho_{J}}\right)+\nu\left(-\frac{1}{r}-\frac{x}{2}-\frac{\sqrt{3} y}{2}+\frac{1}{\rho_{P}}\right)
$$

(because $\rho_{S}=r$ ). For $\mu=\nu=0$ the function $W$ has nonisolated critical points along the circle $\{r=1\}:\left(\frac{\partial W}{\partial r}, \frac{\partial W}{\partial \varphi}\right)=\left(r-\frac{1}{r^{2}}, 0\right)$. Therefore, the genuine critical points should lie near $(r, \varphi)=\left(1, \varphi_{j}\right)$, where $\varphi_{j}$ are critical points of the function

$$
\begin{aligned}
\varphi \longmapsto \mu\left(-\frac{1}{r}-x+\frac{1}{\rho_{J}}\right)+\left.\nu\left(-\frac{1}{r}-\frac{x}{2}-\frac{\sqrt{3} y}{2}+\frac{1}{\rho_{P}}\right)\right|_{r=1} & = \\
& =-\mu-\nu+\mu F(\varphi)+\nu F\left(\frac{\pi}{3}-\varphi\right)
\end{aligned}
$$

where

$$
F(\varphi)=-\cos \varphi+\frac{1}{2|\sin (\varphi / 2)|}
$$

and

$$
F^{\prime}(\varphi)=\sin \varphi \mp \frac{\cos (\varphi / 2)}{4 \sin ^{2}(\varphi / 2)}=\frac{\cos (\varphi / 2)}{4 \sin ^{2}(\varphi / 2)}\left(8 \sin ^{3}(\varphi / 2) \mp 1\right) .
$$

The equation ${ }^{4}$

$$
\mu F^{\prime}(\varphi)-\nu F^{\prime}\left(\frac{\pi}{3}-\varphi\right)=0
$$

for the values $\varphi_{j}$, can have many solutions, which cannot be written explicitly. Instead of solving it for $\varphi$, we solve it rather for $\nu$. Namely, we fix $\varphi=\varphi_{j}$ and then we can define $\nu$ as a function of $\mu$ and $\varphi_{j}$. Concretely, we present this solution in the following parametric form:

$$
\begin{aligned}
& \mu=\delta \cos \theta^{\prime} \sin ^{2} \theta\left(8 \sin ^{3} \theta^{\prime} \mp 1\right), \\
& \nu=\delta \cos \theta \sin ^{2} \theta^{\prime}\left(8 \sin ^{3} \theta \mp 1\right),
\end{aligned}
$$

\footnotetext{
${ }^{4}$ This equation is analogous to equations for positions of $n$ points of small mass near the critical circle presented in [14].
} 
where $\delta$ is a small parameter and

$$
\theta=\frac{\varphi_{j}}{2}, \quad \theta^{\prime}=\frac{\pi}{6}-\frac{\varphi_{j}}{2}
$$

Let us fix such a libration point $L_{\theta}:(r, \varphi) \approx(1,2 \theta)$. We compute the approximate Taylor expansion of $W$ at $L_{\theta}$ up to order 4 . To this end we use Lemma 1 with several variants of $Q$.

For $Q=Q_{S}=\left(x_{S}, y_{S}\right)$, with $\left|Q_{S}\right|=1$, we have

$$
\begin{aligned}
\frac{1}{r}=\frac{1}{\left|Q_{S}+q\right|} & =\text { const }+\operatorname{lin}(q)+\frac{1}{2}\left\{3\left(Q_{S}, q\right)^{2}-|q|^{2}\right\}+ \\
+ & \frac{\left(Q_{S}, q\right)}{2}\left\{3|q|^{2}-5\left(Q_{S}, q\right)^{2}\right\}+\frac{1}{8}\left\{3|q|^{4}-60|q|^{2}\left(Q_{S}, q\right)^{2}+35\left(Q_{S}, q\right)^{4}\right\}+\ldots,
\end{aligned}
$$

where $\operatorname{lin}(q)$ denotes the linear part.

For $Q_{J}=\left|Q_{J}\right| \widetilde{Q}_{J}=\left|Q_{J}\right|\left(\widetilde{x}_{J}, \widetilde{y}_{J}\right)$ we have

$$
\frac{1}{\rho_{J}}=\text { const }+\operatorname{lin}(q)+\frac{1}{2\left|Q_{J}\right|^{3}}\left\{\left(3 \widetilde{Q}_{J}, q\right)^{2}-|q|^{2}\right\}+\ldots,
$$

and for $Q_{P}=\left|Q_{P}\right| \widetilde{Q}_{P}=\left|Q_{P}\right|\left(\widetilde{x}_{P}, \widetilde{y}_{P}\right)$ we have

$$
\frac{1}{\rho_{P}}=\text { const }+\operatorname{lin}(q)+\frac{1}{2\left|Q_{P}\right|^{5}}\left\{3\left(\widetilde{Q}_{P}, q\right)^{2}-|q|^{2}\right\}+\ldots
$$

Therefore, the quadratic part of $W$ at $L_{\theta}$, modulo $O\left(\delta^{2}\right)$, is

$$
\begin{aligned}
W_{2}(q)=\frac{1}{2}|q|^{2}+\frac{1-\mu-\nu}{2}\{ & \left.3\left(Q_{S}, q\right)^{2}-|q|^{2}\right\}+ \\
& +\frac{\mu}{2\left|Q_{J}\right|^{3}}\left\{3\left(\widetilde{Q}_{J}, q\right)^{2}-|q|^{2}\right\}+\frac{\nu}{2\left|Q_{P}\right|^{3}}\left\{3\left(\widetilde{Q}_{P}, q\right)^{2}-|q|^{2}\right\}
\end{aligned}
$$

and hence

$$
\begin{aligned}
& a=3(1-\mu-\nu) x_{S}^{2}+\mu\left\{1+3 \frac{\widetilde{x}_{J}^{2}}{\left|Q_{J}\right|^{3}}-\frac{1}{\left|Q_{J}\right|^{3}}\right\}+\nu\left\{1+3 \frac{\widetilde{x}_{P}^{2}}{\left|Q_{P}\right|^{3}}-\frac{1}{\left|Q_{P}\right|^{3}}\right\}, \\
& b=3(1-\mu-\nu) x_{S} y_{S}+3 \mu \frac{\widetilde{x}_{J} \widetilde{y}_{J}}{\left|Q_{J}\right|^{3}}+3 \nu \frac{\widetilde{x}_{P} \widetilde{y}_{P}}{\left|Q_{P}\right|^{3}} \\
& c=3(1-\mu-\nu) y_{S}^{2}+\mu\left\{1+3 \frac{\widetilde{y}_{J}^{2}}{\left|Q_{J}\right|^{3}}-\frac{1}{\left|Q_{J}\right|^{3}}\right\}+\nu\left\{1+3 \frac{\widetilde{y}_{P}^{2}}{\left|Q_{P}\right|^{3}}-\frac{1}{\left|Q_{P}\right|^{3}}\right\} .
\end{aligned}
$$

Let us check the conditions from Lemma 3 (for linear stability). For $\mu=\nu=0$, i. e., $\delta=0$ in Eqs. (5.5), we have

$$
a_{2}=4-3\left(x_{S}^{2}+y_{S}^{2}\right)=1 .
$$

Next,

$$
\begin{aligned}
a_{4}=\frac{3 \mu}{\left|Q_{J}\right|^{3}}\left\{\left(\left|Q_{J}\right|^{3}-1\right)+3\left(x_{S} \widetilde{y}_{J}-y_{S} \widetilde{x}_{J}\right)^{2}\right\} & + \\
& +\frac{3 \nu}{\left|Q_{P}\right|^{3}}\left\{\left(\left|Q_{P}\right|^{3}-1\right)+3\left(x_{S} \widetilde{y}_{P}-y_{S} \widetilde{x}_{P}\right)^{2}\right\}+\ldots ;
\end{aligned}
$$


so, $\left.a_{4}\right|_{\delta=0}=0$ and $\Delta>0$. Therefore, we should find values of $\theta$ for which $\delta$ can be chosen such that $a_{4}>0$. From Eq. (5.6) we see that this holds when

$$
\mu, \nu>0, \quad\left|Q_{J}\right|,\left|Q_{P}\right|>1
$$

The second condition means that

$$
\frac{2 \pi}{3}<\varphi<\frac{5 \pi}{3}
$$

Note that $F^{\prime}(\varphi)= \pm \cos (\varphi / 2)\left|Q_{J}\right|^{-2}\left(\left|Q_{J}\right|^{3}-1\right)$ (see Eq. (5.4)), where $\pm=+$ for $0<\varphi<\pi$ and - otherwise. Analogously, $F^{\prime}(\pi / 3-\varphi)= \pm \cos (\pi / 6-\varphi / 2)\left|Q_{P}\right|^{-2}\left(\left|Q_{P}\right|^{3}-1\right)$ with analogous choice of signs. So, under the restriction (5.7), the signs of $F^{\prime}(2 \theta)$ and $F^{\prime}\left(2 \theta^{\prime}\right)$ are the same in the domain

$$
-\pi<\varphi_{j}=2 \theta<-\frac{2 \pi}{3}
$$

(opposite to the $\operatorname{arc} J P$ ). Then we can choose $\delta$ in Eq. (5.5) such that $\mu, \nu>0$ (physically sensible choice).

On the other hand, $\mu$ and $\nu$ have the same sign also when $0<\varphi_{j}=2 \theta<\pi / 3$. Noting that $\left|x_{S} \widetilde{y}_{J}-y_{S} \widetilde{x}_{J}\right|=|\cos \theta|$ and $\left|x_{S} \widetilde{y}_{P}-y_{S} \widetilde{x}_{P}\right|=|\cos (\pi / 6-\theta)|$ in Eq. (5.6), we can add the additional arc to (5.8):

$$
\arcsin (1 / 8)<\theta<\frac{\pi}{6}-\arcsin (1 / 8)
$$

(inside the $\operatorname{arc} J P$ ).

Theorem 3. The corresponding equilibrium point of the Hamiltonian system is stable in the Lyapunov sense for typical values of the parameter $\theta$ from the intervals (5.8) and (5.9).

Proof. We know that, if the libration point $L:(r, \varphi) \approx(1,2 \theta)$ is such that inequalities (5.8)-(5.9) are obeyed, then it is linearly stable.

Note that for $\mu=\nu=0$ we have $a_{2}=1$ and $a_{4}=0$, i. e., we deal with the $0: 1$ resonance, with the eigenvalues $\pm i \omega_{1}= \pm i$ and 0 (double). But, unlike in the previous two sections, here we deal with a bifurcation problem depending on the additional parameter $\theta$, which is neither small nor large.

For $\mu=\nu=0$ we have $a=3 x_{S}^{2}, b=3 x_{S} y_{S}$ and $c=3 y_{S}^{2}$, where $x_{S}$ and $y_{S}$ are the coordinates of the position $Q_{S}$ of $A$ with respect to $S$. From Section 2.6 we get formulas for $z$, $u$ and $v$. Note that, for $z=u=0$, Eq. (2.23) gives $q_{2}=-(a / b) q_{1}=-\left(x_{S} / y_{S}\right) q_{1}$ and hence $\left.\left(Q_{S}, q\right)\right|_{z=u=0}=0$. Thus, $\left.H_{3}\right|_{z=u=0} \equiv 0$, as in the previous section. So we should proceed as in Section 4 and we need the whole inverse change.

But we do it for some special choice of the parameter $\theta$; we take it such that $\varphi=2 \theta=\frac{5 \pi}{4}$; thus, $x_{S}=y_{S}=-\frac{1}{2} \sqrt{2}$ and $a=b=c=\frac{3}{2}$.

We skip formulas for $z=z_{1}+z_{2} i$, $u$ and $v$, but recall that $\kappa=\{z, \bar{z}\}=2(a+1) i=5 i$. We only use formulas (2.22)-(2.23) for the inverse change. We have

$$
q_{1}=\frac{4}{3} \widetilde{u}+\widetilde{v}-\frac{4}{5} z_{1}-\frac{3}{5} z_{2}, \quad q_{2}=-\widetilde{v}+z_{2}
$$

where $\widetilde{u}=\sqrt{a} u=\sqrt{3 / 2} u$ and $\widetilde{v}=\sqrt{3 / 2} v$. Hence,

$$
\left(Q_{S}, q\right)=-\frac{1}{2} \sqrt{2}\left(q_{1}+q_{2}\right)=\frac{1}{2} \sqrt{2}\left(\frac{4}{3} \widetilde{u}-\frac{4}{5} z_{1}+\frac{2}{5} z_{2}\right)
$$




$$
\begin{aligned}
|q|^{2} & =\left(\frac{4}{3} \widetilde{u}+\widetilde{v}-\frac{4}{5} z_{1}-\frac{3}{5} z_{2}\right)^{2}+\left(-\widetilde{v}+z_{2}\right)^{2}= \\
& =\frac{16}{9} \widetilde{u}^{2}+\frac{8}{3} \widetilde{u} v-\frac{32}{15} \widetilde{u} z_{1}-\frac{8}{5} \widetilde{u} z_{2}+2 \widetilde{v}^{2}-\frac{8}{5} \widetilde{v} z_{1}-\frac{16}{5} \widetilde{v} z_{2}+\frac{16}{25} z_{1}^{2}+\frac{24}{25} z_{1} z_{2}+\frac{34}{25} z_{2}^{2} .
\end{aligned}
$$

We have $Q_{J}=\frac{-1}{\sqrt{2}}(1+\sqrt{2}, 1) \approx-(1.7071,0.7071)$ with $\left|Q_{J}\right| \approx 1.8478, Q_{P}=$ $=\frac{-1}{\sqrt{2}}(1+\sqrt{2}, \sqrt{2}+\sqrt{3}) \approx-(1.7071,2.2247)$ with $\left|Q_{P}\right| \approx 2.8042, \theta=\frac{5}{8} \pi, \theta^{\prime}=\frac{13}{23} \pi$. This gives

$$
\begin{aligned}
& \mu=-\frac{1}{4}\left|Q_{J}\right|^{2} \cos \theta^{\prime}\left(\left|Q_{P}\right|^{3}-1\right) \delta=3.6559 \cdot \delta, \\
& \nu=-\frac{1}{4}\left|Q_{P}\right|^{2} \cos \theta\left(\left|Q_{J}\right|^{3}-1\right) \delta=3.9941 \cdot \delta,
\end{aligned}
$$

where $\delta>0$ and small. Next, we find

$$
a \approx \frac{3}{2}+2.0640 \cdot \delta, \quad b \approx \frac{3}{2}-7.3129 \cdot \delta, \quad c \approx \frac{3}{2}-1.027 \cdot \delta .
$$

Therefore, for $z=u=0$, we have $\left.H_{2} \approx H_{2}\right|_{\delta=0}+\frac{1}{2} \cdot 5.2209 \cdot \delta v^{2}$; we put $\varepsilon \approx-5.2209 \cdot \delta$.

Next, $H_{3}$, whose main contribution comes from $-1 / \rho_{S}=-1 / r$, equals

$$
\begin{aligned}
& H_{3} \approx- \frac{1}{2}\left(Q_{S}, q\right)\left\{3|q|^{2}-5\left(Q_{S}, q\right)^{2}\right\} \\
&=-2 \sqrt{2} \widetilde{u} \widetilde{v}^{2}+\frac{6}{5} \sqrt{2} \widetilde{v}^{2} z_{1}-\frac{3}{5} \sqrt{2} \widetilde{v}^{2} z_{2}-\frac{24}{25} \sqrt{2} \widetilde{v} z_{1}^{2}-\frac{36}{25} \sqrt{2} \widetilde{v} z_{1} z_{2}+\frac{24}{25} \sqrt{2} \widetilde{v} z_{2}^{2}= \\
&=0 \cdot v^{3}+0 \cdot v|z|^{2}-3 \sqrt{3} u v^{2}+\left[\frac{9}{10} \sqrt{2}\left(1+\frac{i}{2}\right) v^{2} z+\text { conj }\right]
\end{aligned}
$$

plus inessential terms. By Eq. (2.11) this gives

$$
A_{1}=A_{2}=0, \quad k_{12}=-3 \sqrt{3}, \quad m_{02}=\frac{9}{10} \sqrt{2}\left(1+\frac{1}{2} i\right) .
$$

Next,

$$
\left.H_{4}\right|_{z=u=0}=-\frac{3}{8}|q|^{4}=-v^{4},
$$

which gives $A_{3}=-1$. In Eq. (2.13), for small $\varepsilon$, we get

$$
\begin{gathered}
B_{1}=O(\varepsilon), \quad B_{2}=O(\varepsilon), \quad B_{4}=O(1), \\
B_{5}=O(1), \quad B_{3} \approx \frac{2419}{200} .
\end{gathered}
$$

As in the previous section, we find that the main contribution to det comes from

$$
D_{22} \approx-\frac{3 B_{3}}{2 \varepsilon} \neq 0 ;
$$

hence, det $\approx-2 \omega_{1}^{2} D_{22} \not \equiv 0$. 


\section{References}

[1] Arenstorf, R.F., Central Configurations of Four Bodies with One Inferior Mass, Celestial Mech., 1982, vol. 28, nos. 1-2, pp. 9-15.

[2] Arnol'd, V. I., Mathematical Methods of Classical Mechanics, 2nd ed., Grad. Texts in Math., vol. 60, New York: Springer, 1997.

[3] Arnol'd, V.I., Kozlov, V.V., and Neřshtadt, A. I., Mathematical Aspects of Classical and Celestial Mechanics, 3rd ed., Encyclopaedia Math. Sci., vol. 3, Berlin: Springer, 2006.

[4] Baltagiannis, A. N. and Papadakis, K. E., Equilibrium Points and Their Stability in the Restricted Four-Body Problem, Internat. J. Bifur. Chaos Appl. Sci. Engrg., 2011, vol. 21, no. 8, pp. 2179-2193.

[5] Baltaggianis, A. N. and Papadakis, K. N., Families of Periodic Orbits in the Restricted Four-Body Problem, Astrophys. Space Sci., 2011, vol. 336, no. 2, pp. 357-367.

[6] Bardin, B.S., On Motions near the Lagrange Equilibrium Point $L_{4}$ in the Case of Routh's Critical Mass Ratio, Celest. Mech. Dynam. Astronom., 2002, vol. 82, no. 2, pp. 163-177.

[7] Bardin, B.S. and Esipov, P. A., Investigation of Lyapunov Stability of a Central Configuration in the Restricted Four-Body Problem, AIP Conf. Proc., 2018, vol. 1959, no. 1, Art. 040004.

[8] Barwicz, W., Wiliński, M., and Żołądek, H., Birkhoff Normalization, Bifurcations of Hamiltonian Vector Fields and the Deprits Formula, J. Fixed Point Theory Appl., 2013, vol. 13, pp. 587-610.

[9] Birkhoff, G. D., Dynamical Systems, Amer. Math. Soc. Colloq. Publ., vol. 9, Providence, R.I.: AMS, 1966.

[10] Brumberg, V.A., Permanent Configurations in the Problem of Four Bodies and Their Stability, Soviet Astronomy (A. J.), 1957, vol. 1, no. 1, pp. 57-79; see also: Astron. Zh., 1957, vol. 34, no. 1, pp. 55-74.

[11] Broer, H., Hoveijn, I., Lunter, G., and Vegter, V., Bifurcations in Hamiltonian Systems: Computing Singularities by Gröbner Bases, Lect. Notes Math., vol. 1806, Berlin: Springer, 2003.

[12] Bud'ko, D. A. and Prokopenya, A. N., Symbolic-Numerical Analysis of Equilibrium Solutions in a Restricted Four-Body Problem, Program. Comput. Softw., 2010, vol. 36, no. 2, pp. 68-74; see also: Programmirovanie, 2010, vol. 36, no. 2, pp. 13-20.

[13] Burgos-García, J. and Gidea, M., Hill's Approximation in a Restricted Four-Body Problem, Celestial Mech. Dynam. Astronom., 2015, vol. 122, no. 2, pp. 117-141.

[14] Corbera, M., Cors, J. M., and Llibre, J., On the Central Configurations of the Planar $1+3$ Body Problem, Celestial Mech. Dynam. Astronom., 2011, vol. 109, no. 1, pp. 7-43.

[15] Deprit, A. and Deprit-Bartholomé, A., Stability of the Triangular Lagrangian Points, Astron. J., 1967, vol. 72, no. 2, pp. 173-179.

[16] Duistermaat, J. J., The Monodromy in the Hamiltonian Hopf Bifurcation, Z. Angew. Math. Phys., 1998, vol. 49, no. 1, pp. 156-161.

[17] Giorgilli, A., Delshams, A., Fontich, E., Galgani, L., and Simó, C., Effective Stability for a Hamiltonian System near an Elliptic Equilibrium Point, with an Application to the Restricted Three-Body Problem, J. Differential Equations, 1989, vol. 77, no. 1, pp. 167-198.

[18] Grebenikov, E.A., Kozak-Skoworodkina, D., and Jakubiak, M., Computer Algebra Methods in the Many-Body Problem, Moskow: RUDN, 2001 (Russian).

[19] Howell, K. C. and Spencer, D. B., Periodic Orbits in the Restricted Four-Body Problem, Acta Astronaut., 1986, vol. 13, no. 8, pp. 473-479.

[20] Leandro, E. S. G., On the Central Configurations of the Planar Restricted Four-Body Problem, J. Differential Equations, 2006, vol. 226, no. 1, pp. 323-351.

[21] Leontovich, A. M., On Stability of the Lagrangian Periodic Solutions of the Restricted Three-Body Problem, Dokl. Akad. Nauk SSSR, 1962, vol. 143, no. 3, pp. 525-528 (Russian).

[22] Liu, B. and Zhou, Q., Linear Stability of Elliptic Relative Equilibria of Restricted Four-Body Problem, J. Differential Equations, 2020, vol. 269, no. 6, pp. 4751-4798. 
[23] Markeev, A. P., Libration Points in Celestial Mechanics and Space Dynamics, Moscow: Nauka, 1978 (Russian).

[24] Markeyev, A. P., The Critical Case of a Pair of Zero Roots in a Two-Degree-of-Freedom Hamiltonian System, J. Appl. Math. Mech., 1998, vol. 62, no. 3, pp. 341-349; see also: Prikl. Mat. Mekh., 1998, vol. 62 , no. 3 , pp. $372-382$.

[25] Markeev, A. P., On Stability and Non-Linear Oscillations of Hamiltonian System in a Resonant Case, Mech. Solids, 1998, vol. 33, no. 4, pp. 32-41; see also: Izv. Ross. Akad. Nauk. Mekh. Tverd. Tela, 1998, no. 4, pp. 38-49.

[26] Markeyev, A. P., Non-Linear Oscillations of a Hamiltonian System with $2: 1$ Resonance, J. Appl. Math. Mech., 1999, vol. 63, no. 5, pp. 715-726; see also: Prikl. Mat. Mekh., 1999, vol. 63, no. 5, pp. $757-769$.

[27] Markeyev, A. P., Non-Linear Oscillations of a 1:1 Resonance Hamiltonian System, J. Appl. Math. Mech., 2011, vol. 75, no. 6, pp. 631-646; see also: Prikl. Mat. Mekh., 2011, vol. 75, no. 6, pp. 901-922.

[28] Moser, J. K., Lectures on Hamiltonian Systems, Mem. Amer. Math. Soc., vol. 81, Providence, R.I.: AMS, 1968.

[29] Prokopenya, A. N., Hamiltonian Normalization in the Restricted Many-Body Problems by Computer Algebra Methods, Program. Comput. Softw., 2012, vol. 38, no. 3, pp. 156-166; see also: Programmirovanie, 2012, vol. 38, no. 3, pp. 65-78.

[30] Siegel, C.L. and Moser, J. K., Lectures on Celestial Mechanics, Grundlehren Math. Wiss., vol.187, New York: Springer, 1971.

[31] van der Meer, J.-C., Bifurcation at Non-Semisimple 1:-1 Resonance, J. Appl. Math. Phys., 1986, vol. 37 , pp. $425-437$. 